then performed x-ray diffraction measurements and molecular modeling to demonstrate that the -3P SAM matched the molecules' orientation and the packing of the nascent anthracene crystal in the $c^{*}$-direction. Thus -3P SAMs are a nearly perfect template for the oriented nucleation of the anthracene crystals from the (001) plane. On the other hand, $-\mathrm{CH}_{3}$ SAMs have the least nucleation density of anthracene crystals and the least surface coverage.

The researchers then addressed the possibility of controlling the location and the pattern of nucleation. To achieve a better selectivity of patterned growth, they designed the template with SAMs of two extremes bearing $-\mathrm{CH}_{3}$ and $-3 \mathrm{P}$. The research team used the microcontact printing $(\mu \mathrm{CP})$ method to make micropatterned substrates with regions of $-3 \mathrm{P}$ and $-\mathrm{CH}_{3}$ having different geometries and relative sizes. The team used both solvent evaporation and dip-coating methods to achieve selective growth of organic semiconductor molecules. The researchers observed highly localized crystal growth onto regions patterned with oligophenylene thiols. The surface coverage of crystals grown on micropatterned $-3 \mathrm{P}$ regions increased to $>95 \%$ versus $23 \%$ for the unpatterned $-3 \mathrm{P}$ substrates. Using this method, the researchers obtained large oriented single crystals of anthracene overgrowing the entire underlying $100 \mu \mathrm{m} \times 100 \mu \mathrm{m}$ square regions of $-3 \mathrm{P}$. The researchers said that the flux of molecules to the growing crystals in the $-3 \mathrm{P}$ region will induce near-surface concentration gradients and deplete anthracene molecules from the less active alkylthiol areas. This is a promising way to achieve highly selective patterned growth of single-crystalline organic semiconductor with increased surface coverage, they said.

\section{Light-Induced Effects Dominate Transconductance in OFETs}

The physics of photoinduced charge transfer across organic interfaces in organic field-effect transistors (OFETs) is not well known, but is crucial to the development of devices such as active-matrix displays. Two researchers, V. Podzorov and M.E. Gershenson, from Rutgers University have now published their results for a mechanism of photoinduced charge transfer across the interface between an organic semiconductor (rubrene) and a polymer (parylene). In their article in the July 1 issue of Physical Review Letters (\#016602), the researchers said that their discovery could be crucial for understanding optoelectronic devices such as active-matrix displays. They carried out their experiments on single-crystal rubrene OFETs with parylene as the gate dielectric. The researchers said that the frontgate FET design with a transparent dielectric and semitransparent gate electrode allows them to study the light-induced effects at the organic molecular crystal (OMC)-polymer interface over a wide spectral range that includes the absorption band of OMC.

In the dark, the "as-prepared" single-crystal rubrene OFETs exhibit a very small field-effect threshold. The researchers said this indicates that the density of deep traps at the OMC-parylene interface does not exceed $10^{10} \mathrm{~cm}^{-2}$. Prolonged application of the gate voltage $\left(V_{\mathrm{g}}\right)$ in the dark does not affect the dependence of the source-drain current $\left(I_{\mathrm{SD}}\right)$ on the gate voltage $\left(V_{\mathrm{g}}\right)$, (i.e., the transconductance), they said. However, illumination of the OFETs with a visible light at a fixed gate voltage results in a shift of the dark transconductance characteristics toward lower or higher $V_{\mathrm{g}}$. The shift is characterized by the magnitude of the field-effect onset voltage $\left(V_{\text {onset }}\right)$. The photoinduced $V_{\text {onset }}$ shift is preserved in the dark for days regardless of further measurements at different $V_{\mathrm{g}}$. The researchers said that the observed photoinduced shift of $V_{\text {onset }}$ is caused by the charging of the gate dielectric with the nonequilibrium carriers, photogenerated in the rubrene near the interface, and transferred into the polymer.

The researchers concluded that two conditions are required for the charge transfer: photoexcitation with a photon energy greater than the highest occupied molecular orbital-lowest unoccupied
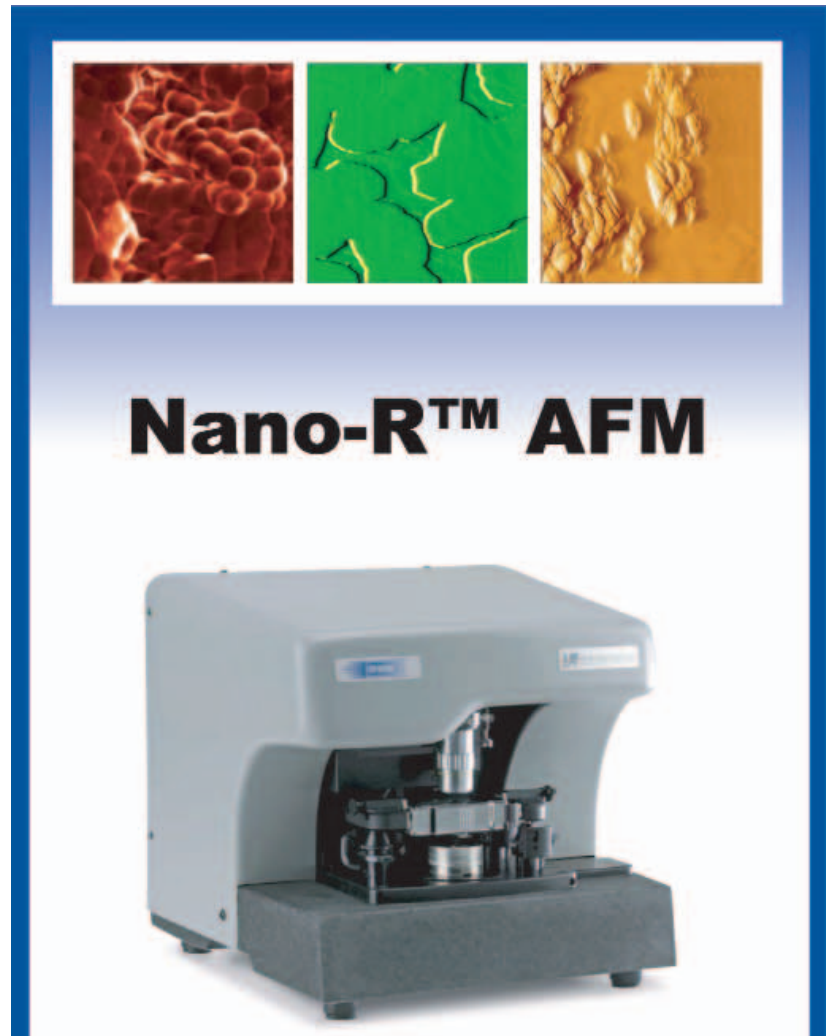

- High Performance

- Easy to Use

- Versatile

Nano- $\mathbf{R}^{\mathrm{TM}}$ is available with a traditional light lever scanner and the advanced Crystal Scanner ${ }^{\mathrm{TM}}$.

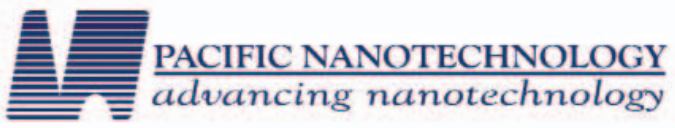

3350 Scott Blvd. \#29

Santa Clara, CA 95054

408-982-9492

www.pacificnanotech.com www.probestore.com
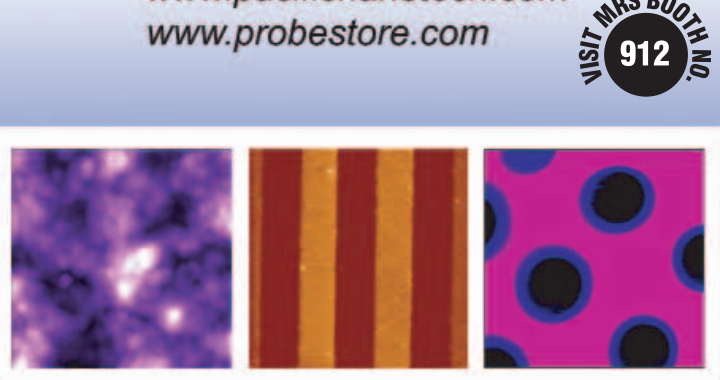

For more information, see http://advertisers.mrs.org 
molecular orbital gap of the organic semiconductor and a transverse electric field applied at the semiconductor-dielectric interface. Trapping of the transferred charge in the polymer results in a controllable and stable-in-the-dark shift of the field-effect threshold, they said.

According to the researchers, the observed effect outlines new aspects of stability of organic devices and opens new possibilities for "programming" OFET characteristics and lithography-free patterning of the conduction channel with light.

VIVEK RANJAN

\section{Liquid Changes to Gel on Demand}

Twisted nanostructures are an important biological motif, such as the DNA double helix or proteins with helical sections important to their function. Artificial helices would be useful in nanotechnological applications. A team of researchers headed by Myongsoo Lee at Yonsei University in Seoul, Korea, has now created a molecular system that can form helices "on demand," turning the initially liquid solution into a gel.

As reported in the September 12 issue of Angewandte Chemie International Edition (p. 5810; DOI: 10.1002/anie.200501270), the researchers developed, as the basic building block for their helices, a molecule with a base consisting of three aromatic rings which is bent like a boomerang. The central ring has a long, branched side chain hanging from it. When a silver salt is added to a solution of these molecules, complexes form between the molecules and the positively charged silver ions; the "boomerangs" latch onto the silver ions. If the negatively charged counterion in the silver salt is boron tetrafluoride $\left(\mathrm{BF}_{4}^{-}\right)$, the complexes pile up into long, twisted columns. The $\mathrm{BF}_{4}^{-}$ions fit into the cavity that remains inside the "belly" of the helices and stabilize them, turning the liquid into a jellylike mass. Upon further study, the researchers found that the helices aggregate into regular bundles of fibers that tangle to form an interwoven, threedimensional network. The liquid trapped inside this fibrous framework forms into a gel. If a fluoride salt is then added to the gel, it liquefies. This is a result of the attraction of the fluoride ions $\left(\mathrm{F}^{-}\right)$for the silver ions, which are lured out of their complexes. The fibrous aggregates collapse back into individual molecules. This effect is reversible if the fluoride ions are trapped by the addition of other salts.

If salts containing $\mathrm{C}_{2} \mathrm{~F}_{5} \mathrm{CO}_{2}{ }^{-}$ions are added to the gel, it also liquefies. Electron microscopy images show that in this case, the phenomenon has a different cause. The complexes do not fall apart into individual molecules, but form individual zigzagging bands. The reason for this change in structure is the difference in size of the anions: $\mathrm{C}_{2} \mathrm{~F}_{5} \mathrm{CO}_{2}{ }^{-}$is larger than $\mathrm{BF}_{4}{ }^{-}$and does not fit into the cavity inside the helices; therefore, the helices are not stabilized. The result, said the researchers, is an "intelligent" nanomaterial whose properties can be switched solely by the choice of counterion.

\section{News of MRS Members/Materials Researchers}

André-Jacques Auberton-Hervé, cofounder, president, and CEO of Soitec, has been appointed to SEMI's International
Board of Directors, serving as the association's European representative.

Keith Bowen, director of technology at
Bede, Durham, U.K., and Brian Tanner, co-founder of Bede and professor of physics at the University of Durham,

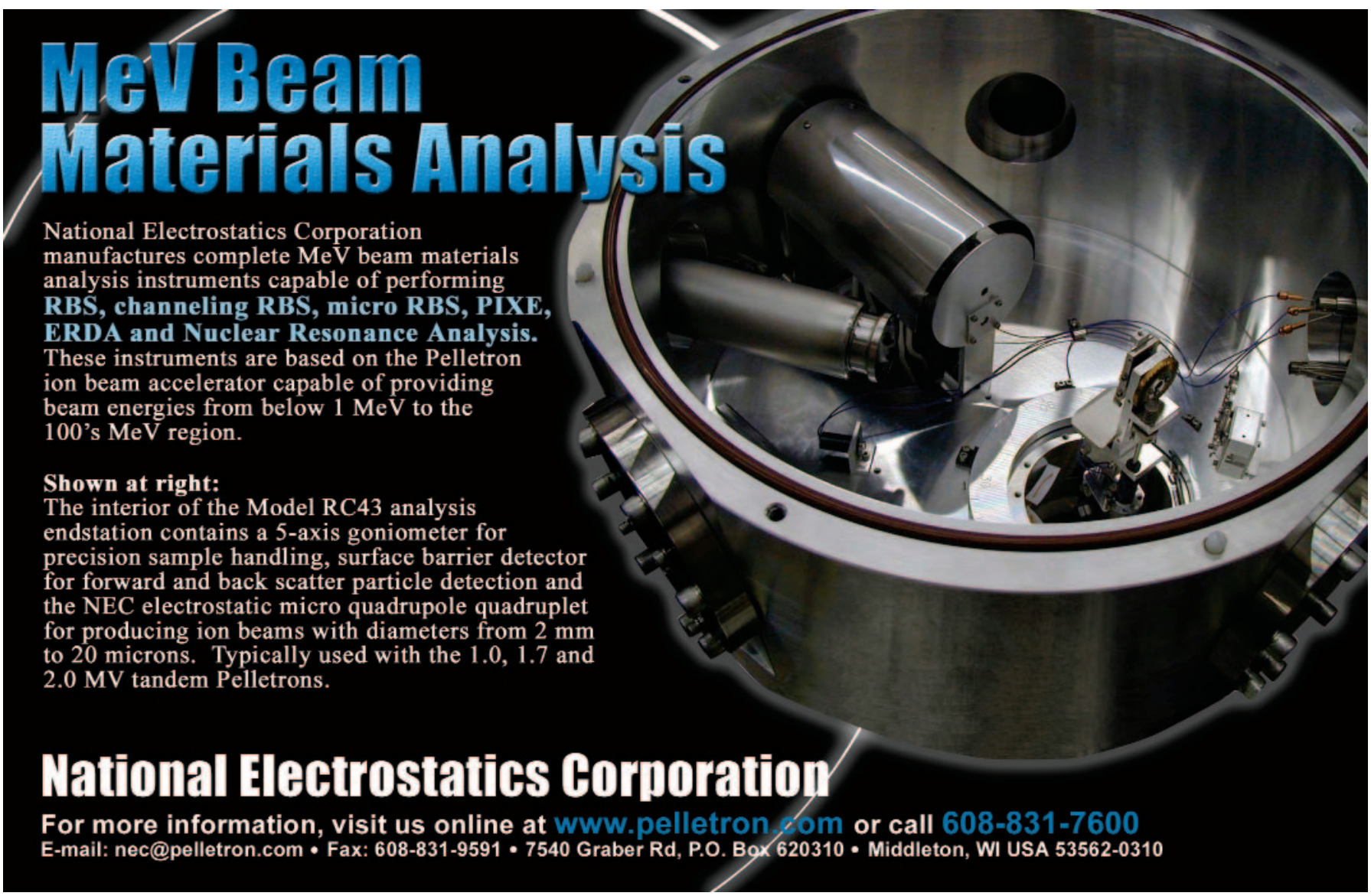

For more information, see http://advertisers.mrs.org 